\title{
A STRONGLY NONLINEAR PROBLEM ARISING IN GLACIOLOGY
}

\author{
JACQUES COLINGE $^{1}$ AND JACQUES RAPPAZ ${ }^{2}$
}

\begin{abstract}
The computation of glacier movements leads to a system of nonlinear partial differential equations. The existence and uniqueness of a weak solution is established by using the calculus of variations. A discretization by the finite element method is done. The solution of the discrete problem is proved to be convergent to the exact solution. A first simple numerical algorithm is proposed and its convergence numerically studied.

Résumé. La simulation des mouvements d'un glacier conduit à la résolution numérique d'un système d'équations aux dérivées partielles fortement non-linéaire. Dans cet article nous prouvons l'existence et l'unicité d'une solution faible de ce système en utilisant des éléments de calcul des variations. Après avoir établi une discrétisation du problème par la méthode des éléments finis, nous montrons que le problème discret a une solution unique qui converge vers la solution exacte. Nous proposons un algorithme de résolution dont nous étudions numériquement la convergence.
\end{abstract}

AMS Subject Classification. 35J60, 65N30, 26B25, 35J20, 49J45, 86A40.

Presented June 10, 1998 by J. Rappaz, member of the Editorial Board.

\section{THE MODEL}

In order to conduct theoretical studies, glaciologists often consider the so-called infinite parallel sided slab, see [1]. This idealised glacier is made of an infinite ice mass situated between two parallel planes with a slight inclination; ice is treated as an incompressible viscous fluid. In this paper we limit our study to the 2-dimensional first order model (Blatter [1]) in a finite domain, i.e. a rectangle.

Let $\Lambda=(0, L) \times(0,1) \ni(x, z), L>0$, be the domain, the boundary of which is denoted by $\partial \Lambda$. The system of equations to solve in $\Lambda$ is

$$
\begin{aligned}
& \frac{\partial \tau}{\partial z}=-2 \frac{\partial \sigma}{\partial x}-1, \\
& \frac{\partial w}{\partial z}=-F(\sigma, \tau) \sigma \\
& \frac{\partial u}{\partial z}=2 F(\sigma, \tau) \tau \\
& \frac{\partial u}{\partial x}=F(\sigma, \tau) \sigma .
\end{aligned}
$$

Keywords and phrases. Nonlinear problem, finite elements, convex analysis, calculus of variations, glaciology.

1 Section of Mathematics, Université de Genève, Rue du Lièvre 2-4, 1227 Acacias, Suisse.

2 Department of Mathematics, École Polytechnique Fédérale de Lausanne, 1015 Lausanne, Suisse.

e-mail: jacques.rappaz@epfl.ch 
The variables have the following meaning: $x$ and $z$ are the Cartesian coordinates, $u$ and $w$ stand for the horizontal and vertical velocities respectively, $\tau$ stands for the shear stress and $\sigma$ stands for the $x$-component of the deviatoric stress tensor. The function $F(\sigma, \tau)$ represents a flow law; it is related to the inverse of the viscosity. We consider an often used law in glaciology, i.e. Glen's flow law

$$
F(\sigma, \tau)=\left(T_{0}^{2}+\tau^{2}+\sigma^{2}\right)^{\frac{n-1}{2}}
$$

where $T_{0} \neq 0$ is a real constant and $n$ is a positive integer.

The boundary conditions are

$$
\begin{aligned}
u(x, z) & =g(x, z), \quad(x, z) \in \Gamma_{0} \\
\tau(x, z) & =0, \quad(x, z) \in \Gamma_{1}, \\
w(x, 0) & =0 .
\end{aligned}
$$

The boundary subset $\Gamma_{1}$ contains the ice mass surface, i.e. $(0, L) \times\{1\} \subset \Gamma_{1}$, but it does not contain the left and right sides, i.e. $\{0\} \times[0,1] \cap \Gamma_{1}=\emptyset$ and $\{L\} \times[0,1] \cap \Gamma_{1}=\emptyset$; we define $\Gamma_{0}=\partial \Lambda \backslash \Gamma_{1}$. The places where the ice can move freely are represented by $\Gamma_{1}$. The function $g$ is given; it represents the imposed velocity on the left and right sides and at some places of the ice mass base.

\section{ANALYsis}

Equation (2) for $w$ can be left aside for the moment, because $w$ can be computed when $\tau$ and $\sigma$ are known. We transform the equations in a more convenient form. We have $F(\sigma, \tau)>0$ and from equations (3) and (4) we obtain

$$
\begin{aligned}
\tau & =\frac{1}{2 F(\sigma, \tau)} \frac{\partial u}{\partial z} \\
\sigma & =\frac{1}{F(\sigma, \tau)} \frac{\partial u}{\partial x}
\end{aligned}
$$

With equation (1) we find

$$
\frac{\partial}{\partial z}\left(\frac{1}{2 F(\sigma, \tau)} \frac{\partial u}{\partial z}\right)+2 \frac{\partial}{\partial x}\left(\frac{1}{F(\sigma, \tau)} \frac{\partial u}{\partial x}\right)=-1
$$

After the coordinate transformation $y=2 z, v(x, y)=u(x, y / 2)$, we have to solve the system

$$
\begin{aligned}
\tau & =\frac{1}{F(\sigma, \tau)} \frac{\partial v}{\partial y} \\
\sigma & =\frac{1}{F(\sigma, \tau)} \frac{\partial v}{\partial x} \\
\frac{1}{2} & =-\frac{\partial}{\partial y}\left(\frac{1}{F(\sigma, \tau)} \frac{\partial v}{\partial y}\right)-\frac{\partial}{\partial x}\left(\frac{1}{F(\sigma, \tau)} \frac{\partial v}{\partial x}\right)
\end{aligned}
$$

in the new domain $\Omega=(0, L) \times(0,2)$. The boundary conditions are transformed in a straightforward manner for obtaining $v(x, y)=g(x, y / 2), \forall(x, y) \in \Gamma, \frac{\partial u}{\partial \nu}=0$ on $\partial \Omega \backslash \Gamma$, where $\Gamma=\left\{(x, 2 z) ;(x, z) \in \Gamma_{0}\right\}$.

If $n=1$ then $F(\sigma, \tau) \equiv 1$ and the problem is equivalent to $-\Delta v=1 / 2$, with mixed Neumann-Dirichlet boundary conditions. This is a well-posed linear problem with solution in $W^{1,2}(\Omega)=H^{1}(\Omega)$. In the sequel we suppose $n \geq 2$. 
We have the relation

$$
F(\sigma, \tau)=\left(T_{0}^{2}+\sigma^{2}+\tau^{2}\right)^{\frac{n-1}{2}}=\left(T_{0}^{2}+\frac{1}{F^{2}(\sigma, \tau)}|\nabla v|^{2}\right)^{\frac{n-1}{2}} .
$$

We show that $F(\sigma, \tau)$ can be expressed as a function of $|\nabla v|^{2}$. We define $f=F(\sigma, \tau)$ and $s=|\nabla v|^{2}$ and have

$$
f^{\frac{2}{n-1}}-T_{0}^{2}=\frac{s}{f^{2}}
$$

Lemma 1. For all $s \in \mathbb{R}^{+}$there exists a unique $f \in \mathbb{R}^{+}$satisfying (14), which we denote by $f=f(s)$. Moreover there exist two positive constants $\beta$ and $\gamma$ (depending on $n$ ) such that

$$
s^{\frac{n-1}{2 n}} \leq f(s) \leq \gamma(\beta+s)^{\frac{n-1}{2 n}}, s \geq 0 .
$$

Proof. Let $s$ be a non-negative number and set $R(f)=f^{\frac{2}{n-1}}-T_{0}^{2}$ and $T_{s}(f)=\frac{s}{f^{2}}$. Clearly (14) is equivalent to $R(f)=T_{s}(f)$. If $s=0$ we obtain $f=f(0)=\left|T_{0}\right|^{n-1}$. Assume now that $s$ is positive. The function $R$ is strictly increasing with $R(0)=-T_{0}^{2}<0$ and $\lim _{f \rightarrow \infty} R(f)=+\infty$. At the opposite, the function $T_{s}$ is strictly decreasing with $T_{s}(+0)=+\infty$ and $\lim _{f \rightarrow \infty} T_{s}(f)=0$. By using Bolzano's Theorem, we easily conclude for the existence and uniqueness of $f(s)$.

Now we show the estimate (15). By using (14) we have

$$
f^{\frac{2 n}{n-1}}-T_{0}^{2} f^{2}=s
$$

By derivating (16) we obtain, since $f$ is strictly positive,

$$
\left(\frac{2 n}{n-1} f^{\frac{2 n}{n-1}}(s)-2 T_{0}^{2} f^{2}(s)\right) \frac{f^{\prime}(s)}{f(s)}=1
$$

or equivalently

$$
\left(\frac{2}{n-1} f^{\frac{2 n}{n-1}}(s)+2 s\right) \frac{f^{\prime}(s)}{f(s)}=1
$$

Relation (17) proves that $f^{\prime}(s)$ is strictly positive ( $f$ is strictly increasing) and with (16) we obtain $\lim _{s \rightarrow \infty} f(s)=$ $+\infty$. Since $\frac{2 n}{n-1}>2$, it is easy to see from (16) that there exist two positive constants $\gamma$ and $S$ such that

$$
f(s) \leq \gamma s^{\frac{n-1}{2 n}}, \forall s \geq S .
$$

For $s \in[0, S]$, the function $f(s)$ is bounded and we set

$$
\beta=\left(\frac{\max _{s \in[0, S]} f(s)}{\gamma}\right)^{\frac{2 n}{n-1}} .
$$

It follows that

$$
f(s) \leq \gamma(\beta+s)^{\frac{n-1}{2 n}}, \forall s \in \mathbb{R}^{+} .
$$

Finally, from (16) we obtain

$$
f^{\frac{2 n}{n-1}}(s) \geq s
$$

and Lemma 1 is proved. 
If we return to equation (12) we have, by using (13), the formal expression

$$
-\operatorname{div}\left(\frac{1}{f\left(|\nabla v|^{2}\right)} \nabla v\right)=\frac{1}{2}, \text { in } \Omega
$$

Moreover if we define $k(x, y)=g(x, y / 2), \forall(x, y) \in \Gamma$, we obtain the following boundary conditions on $v$

$$
\begin{aligned}
v(x, y) & =k(x, y), \quad \forall(x, y) \in \Gamma, \\
\frac{\partial v}{\partial y}(x, y) & =0, \quad \forall(x, y) \in \partial \Omega \backslash \Gamma .
\end{aligned}
$$

From now on, we assume that $k$ belongs to $W^{1-\frac{1}{p}, p}(\Gamma)$, where $p$ is defined by

$$
p=\frac{n+1}{n} \text {. }
$$

In order to analyse Problems (18-20), we use the affine space

$$
V_{k}=\left\{u \in W^{1, p}(\Omega) ;\left.u\right|_{\Gamma}=k\right\} .
$$

The affine space $V_{k}$ is well defined because the functions belonging to $W^{1, p}(\Omega)$ possess a trace on $\Gamma$ which is in $W^{1-\frac{1}{p}, p}(\Gamma)$ (see [8] for instance). In a similar way we denote by $V_{0}$ the linear space

$$
V_{0}=\left\{u \in W^{1, p}(\Omega) ;\left.u\right|_{\Gamma}=0\right\} .
$$

Let $p^{\prime}$ be the conjugate integer of $p$, i.e. $\frac{1}{p}+\frac{1}{p^{\prime}}=1$. Equation (21) immediately yields $p^{\prime}=n+1$. Now if we take a function $u$ in $W^{1, p}(\Omega)$, we easily verify, by using Lemma 1 , that $\frac{1}{f\left(|\nabla u|^{2}\right)}|\nabla u|$ belongs to $L^{n+1}(\Omega)$. Consequently, Hölder's Inequality allows us to write a weak formulation of Problems (18-20) as follows: Find $v \in V_{k}$ such that

$$
\int_{\Omega} \frac{1}{f\left(|\nabla v|^{2}\right)} \nabla v \nabla w \mathrm{~d} x \mathrm{~d} y=\frac{1}{2} \int_{\Omega} w \mathrm{~d} x \mathrm{~d} y, \quad \forall w \in V_{0}
$$

Let $h(s)=\frac{1}{f(s)}$ and $H(s)=\int_{0}^{s} h(t) \mathrm{d} t$. Lemma 1 implies $H(s) \leq \frac{2 n}{n+1} s^{\frac{n+1}{2 n}}$ and if $u \in W^{1, p}(\Omega)$, then $H\left(|\nabla u|^{2}\right) \in$ $L^{1}(\Omega)$. Therefore the following minimization problem possesses a meaning: Find $v \in V_{k}$ such that

$$
J(v) \leq J(u), \quad \forall u \in V_{k},
$$

where $J$ is defined on $V_{k}$ by

$$
J(u)=\frac{1}{2} \int_{\Omega}\left(H\left(|\nabla u|^{2}\right)-u\right) \mathrm{d} x \mathrm{~d} y .
$$

We show below that the minimization problem (23) has a unique solution which is just the unique solution to the variational problem (22). To aim at this we use the so-called direct methods from the calculus of variations, see e.g. [6]. We denote by

$$
\varphi(u, \xi)=H\left(|\xi|^{2}\right)-u
$$

the integrand of (24); it takes its source in $\mathbb{R} \times \mathbb{R}^{2}$. We observe that $\varphi(u, \xi)$ is continuous. 
Lemma 2. $\varphi(u, \cdot)$ is strictly convex.

Proof. We show that $l(s)=H\left(s^{2}\right), s \geq 0$, is strictly convex. We have

$$
\begin{aligned}
l^{\prime}(s) & =2 s H^{\prime}\left(s^{2}\right)=\frac{2 s}{f\left(s^{2}\right)} \\
l^{\prime \prime}(s) & =\frac{2 f\left(s^{2}\right)-4 s^{2} f^{\prime}\left(s^{2}\right)}{f^{2}\left(s^{2}\right)} .
\end{aligned}
$$

We set $t=s^{2}$ and from

$$
f(t)=\left(T_{0}^{2}+\frac{t}{f^{2}(t)}\right)^{\frac{n-1}{2}}
$$

we deduce

$$
f^{\prime}(t)=\frac{n-1}{2}\left(T_{0}^{2}+\frac{t}{f^{2}(t)}\right)^{\frac{n-3}{2}}\left(\frac{f(t)-2 t f^{\prime}(t)}{f^{3}(t)}\right)
$$

Hence

$$
l^{\prime \prime}(s)=\frac{4}{n-1} f(t) f^{\prime}(t)\left(T_{0}^{2}+\frac{t}{f^{2}(t)}\right)^{\frac{3-n}{2}}
$$

and, since

$$
f^{\prime}(t)=\frac{n-1}{2}\left(T_{0}^{2}+\frac{t}{f^{2}(t)}\right)^{\frac{n-3}{2}} \frac{f(t)}{f^{3}(t)+2 t \frac{n-1}{2}\left(T_{0}^{2}+\frac{t}{f^{2}(t)}\right)^{\frac{n-3}{2}}}>0
$$

we have $l^{\prime \prime}(s)>0$. Therefore we have established the strict convexity of $l(s)$.

The conclusion follows from the fact that $l(s)$ is increasing for positive $s$ : let $\alpha \in(0,1), \xi, \eta \in \mathbb{R}^{2}$, then

$$
l(|\alpha \xi+(1-\alpha) \eta|) \leq l(\alpha|\xi|+(1-\alpha)|\eta|)<\alpha l(|\xi|)+(1-\alpha) l(|\eta|)
$$

Theorem 1. There is a unique $v \in V_{k} \subset W^{1, p}(\Omega)$ such that

$$
J(v)=\inf \left\{J(u) ; u \in V_{k}\right\}<\infty .
$$

Moreover, $v$ is the unique weak solution to (22).

Proof. We follow the approach presented in Dacorogna [6]. There exists $\tilde{k} \in W^{1, p}$ such that $\left.\tilde{k}\right|_{\Gamma}=k$, see Nečas [8]. We clearly have $J(\tilde{k})<\infty$ and then

$$
\inf \left\{J(u) ; u \in V_{k}\right\}=m<\infty \text {. }
$$

Let $\left\{w_{\nu}\right\}_{\nu \in \mathbb{N}}$ be a minimizing sequence for (31). There exists an integer $N$ such that, for all $\nu \geq N$, we have

$$
m+1 \geq J\left(w_{\nu}\right)=\int_{\Omega}\left(H\left(\left|\nabla w_{\nu}\right|^{2}\right)-w_{\nu}\right) \mathrm{d} x \mathrm{~d} y .
$$


In the last part of the proof we introduce several constants, they are always positive but their values can change from one equation to another. Lemma 1 yields

$$
H\left(\left|\nabla w_{\nu}\right|^{2}\right)=\int_{0}^{\left|\nabla w_{\nu}\right|^{2}} \frac{1}{f(s)} \mathrm{d} s \geq c_{1}\left(c_{2}+\left|\nabla w_{\nu}\right|^{2}\right)^{\frac{n+1}{2 n}}-c_{3}
$$

We put (33) into (32) and obtain $\left(p=\frac{n+1}{n}\right)$

$$
\begin{aligned}
m+1 & \geq c_{1} \int_{\Omega}\left(c_{2}+\left|\nabla w_{\nu}\right|^{2}\right)^{\frac{n+1}{2 n}} \mathrm{~d} x \mathrm{~d} y-c_{3} \operatorname{meas}(\Omega)-\int_{\Omega} w_{\nu} \mathrm{d} x \mathrm{~d} y \\
& \geq c_{1} \int_{\Omega}\left|\nabla w_{\nu}\right|^{p} \mathrm{~d} x \mathrm{~d} y-c_{3}-\int_{\Omega} w_{\nu} \mathrm{d} x \mathrm{~d} y \\
& \geq c_{1}\left\|w_{\nu}\right\|_{W^{1, p}}^{p}-c_{3}-\int_{\Omega}\left|w_{\nu}\right| \mathrm{d} x \mathrm{~d} y
\end{aligned}
$$

By using Young's inequality we find

$$
\begin{aligned}
\int_{\Omega}\left|w_{\nu}\right| \mathrm{d} x \mathrm{~d} y & =\int_{\Omega} 1 \cdot\left|w_{\nu}\right| \mathrm{d} x \mathrm{~d} y \leq \int_{\Omega}\left(\frac{1}{p^{\prime} \varepsilon^{p^{\prime}}}+\frac{\varepsilon^{p}}{p}\left|w_{\nu}\right|^{p}\right) \mathrm{d} x \mathrm{~d} y \\
& =\frac{c_{4}}{\varepsilon^{p^{\prime}}} \operatorname{meas}(\Omega)+c_{5} \varepsilon^{p}\left\|w_{\nu}\right\|_{L^{p}}^{p}, \forall \varepsilon>0
\end{aligned}
$$

with $\frac{1}{p}+\frac{1}{p^{\prime}}=1$. Clearly $\left\|w_{\nu}\right\|_{L^{p}}^{p} \leq\left\|w_{\nu}\right\|_{W^{1, p}}^{p}$ and we have

$$
m+1 \geq c_{1}\left\|w_{\nu}\right\|_{W^{1, p}}^{p}-c_{3}-\frac{c_{4}}{\varepsilon^{p^{\prime}}}-\varepsilon^{p} c_{5}\left\|w_{\nu}\right\|_{W^{1, p}}^{p} .
$$

By taking $\varepsilon$ small enough to have $c_{1}-\varepsilon^{p} c_{5}>0$ in (35), we prove that the sequence $\left\{w_{\nu}\right\}_{\nu \in \mathbb{N}}$ is bounded. We can extract a subsequence, still noted $\left\{w_{\nu}\right\}_{\nu \in \mathbb{N}}$, which has a weak limit $v \in V_{k}$.

Lemma 1 easily yields

$$
-|u| \leq \varphi(u, \xi) \leq(c+|u|)\left(1+|\xi|^{p}\right), c>0 .
$$

From Lemma 2 we deduce the convexity of $\varphi(u, \cdot)$ and, with (36), we can apply a theorem found in [7] (Thm. $1.1)$ to prove the weak lower semicontinuity of $J$. So we have

$$
\liminf _{\nu \rightarrow \infty} J\left(w_{\nu}\right) \geq J(v)
$$

and it turns out that $v$ is a minimum. Let us admit the existence of another minimum $v^{\prime}$ and define $\bar{v}=\frac{1}{2}\left(v+v^{\prime}\right)$. The functional $J$ is convex because $\varphi$ is convex; this yields

$$
J(\bar{v}) \leq \frac{1}{2}\left(J(v)+J\left(v^{\prime}\right)\right)=\inf \left\{J(u) ; u \in V_{k}\right\}=m .
$$

Hence $\bar{v}$ is also a solution to the minimization problem. It follows that $J(\bar{v})=\frac{1}{2}\left(J(v)+J\left(v^{\prime}\right)\right)$ and

$$
\int_{\Omega}\left[\frac{1}{2} H\left(|\nabla v|^{2}\right)+\frac{1}{2} H\left(\left|\nabla v^{\prime}\right|^{2}\right)-H\left(\left|\frac{1}{2} \nabla v+\frac{1}{2} \nabla v^{\prime}\right|^{2}\right)\right] \mathrm{d} x \mathrm{~d} y=0 .
$$


If we suppose $v$ and $v^{\prime}$ to be different, then we obtain a contradiction with (37) since the strict convexity of $H(u, \cdot)$, see Lemma 2, implies

$$
\int_{\Omega}\left[\frac{1}{2} H\left(|\nabla v|^{2}\right)+\frac{1}{2} H\left(\left|\nabla v^{\prime}\right|^{2}\right)-H\left(\left|\frac{1}{2} \nabla v+\frac{1}{2} \nabla v^{\prime}\right|^{2}\right)\right] \mathrm{d} x \mathrm{~d} y>0 .
$$

It remains to prove that $v$ is a weak solution to (22). A new application of Lemma 1 yields

$$
\left|\operatorname{grad}_{\xi} \varphi\right| \leq c|\xi|^{p-1}
$$

where $c>0$ is a constant. This bound allows us to apply a result found in [6] (Thm. 4.4, Sect. 3) to conclude.

We observe that the techniques used in the proof of Theorem 1 are not restricted to the rectangular domain $\Omega$. In fact, they remain valid for any bounded open subset of $\mathbb{R}^{2}$ with Lipschitz boundary.

\section{Discretization}

We set discrete equivalent problems to Problem (22) and Problem (23) by using triangle finite elements of degree 1 . We denote by $\mathcal{T}_{h}$ a regular triangulation of $\bar{\Omega}$, with triangles $K \in \mathcal{T}_{h}$ and

$$
X_{h}=\left\{w \in \mathcal{C}^{0}(\bar{\Omega}) ;\left.w\right|_{K} \text { is a polynomial of degree at most } 1, \forall K \in \mathcal{T}_{h}\right\} .
$$

Moreover, we assume that for each triangle $K \in \mathcal{T}_{h}, K \cap \Gamma$ is either void or a side of $K$ or a vertex of $K$. If $r_{h}: \mathcal{C}^{0}(\bar{\Omega}) \rightarrow X_{h}$ is the interpolation operator and if we still denote by $k$ a continuous extension of $k$ to $\bar{\Omega}$, we set

$$
\begin{aligned}
& V_{k, h}=\left\{w \in X_{h} ; w=r_{h} k \text { on } \Gamma\right\} \\
& V_{0, h}=\left\{w \in X_{h} ; w=0 \text { on } \Gamma\right\} .
\end{aligned}
$$

We have $V_{k, h}, V_{0, h} \subset H^{1}(\Omega) \subset W^{1, p}(\Omega)$. Therefore the two following discrete problems are meaningful: Variational discrete problem: Find $v_{h} \in V_{k, h}$ such that

$$
\int_{\Omega} \frac{1}{f\left(\left|\nabla v_{h}\right|^{2}\right)} \nabla v_{h} \nabla w_{h} \mathrm{~d} x \mathrm{~d} y=\frac{1}{2} \int_{\Omega} w_{h} \mathrm{~d} x \mathrm{~d} y, \quad \forall w_{h} \in V_{0, h}
$$

Minimization discrete problem: Find $v_{h} \in V_{k, h}$ such that

$$
J\left(v_{h}\right) \leq J\left(u_{h}\right), \quad \forall u_{h} \in V_{k, h} .
$$

By repeating the proof of Theorem 1 almost unchanged we easily prove the following result:

Theorem 2. Problem (39) possesses a unique solution $v_{h} \in V_{k, h}$ which is also the unique solution of Problem (38).

In order to prove the convergence of the discrete solution to the exact solution as $\mathcal{T}_{h}$ is refined, we need several preliminary results.

Lemma 3. Let $X$ be a Banach space and $\left\{x_{i}\right\}_{i \in \mathbb{N}} \subset X$ a sequence such that, for each subsequence $\left\{x_{i_{j}}\right\}_{j \in \mathbb{N}}$ extracted from $\left\{x_{i}\right\}_{i \in \mathbb{N}}$, we can extract a new subsequence $\left\{x_{i_{j_{l}}}\right\}_{l \in \mathbb{N}}$ which always weakly converges to the same weak limit $x \in X$. Then the original sequence $\left\{x_{i}\right\}_{i \in \mathbb{N}}$ weakly converges to $x$. 
Lemma 4. For $f, g \in L^{P}(\Omega)$ the inequality below holds

$$
\left.\int_{\Omega}|| f\right|^{p}-|g|^{p}\left|\mathrm{~d} x \mathrm{~d} y \leq p\||f|+|g|\|_{L^{p}}^{p-1}\|f-g\|_{L^{p}} .\right.
$$

Proof. Let $x, y \in \mathbb{R}$. By the Mean Value Theorem there exists $\xi \in \mathbb{R}$ such that

$$
|y|^{p}-|x|^{p}=p|\xi|^{p-1}(|y|-|x|) .
$$

By taking the absolute value of this expression and bounding $|\xi|$ by $|x|+|y|$ we find

$$
\left.|| y\right|^{p}-|x|^{p}\left|\leq p(|x|+|y|)^{p-1}\right||y|-|x|\left|\leq p(|x|+|y|)^{p-1}\right| y-x \mid .
$$

Now considering the integral we obtain

$$
\left.\int_{\Omega}|| f\right|^{p}-|g|^{p}\left|\mathrm{~d} x \mathrm{~d} y \leq p \int_{\Omega}(|f|+|g|)^{p-1}\right| f-g \mid \mathrm{d} x \mathrm{~d} y .
$$

The harmonic conjugate of $p$ is $q=\frac{p}{p-1}$ and $f, g \in L^{p}(\Omega)$ implies that $|f-g| \in L^{p}(\Omega)$ and $(|f|+|g|)^{p-1} \in L^{q}(\Omega)$. We apply Hölder's Inequality and obtain

$$
p \int_{\Omega}(|f|+|g|)^{p-1}|f-g| \mathrm{d} x \mathrm{~d} y \leq p\||f|+|g|\|_{L^{p}}^{p-1}\|f-g\|_{L^{p}}
$$

Lemma 5. The functional $J$ is continuous in $W^{1, p}(\Omega)$.

Proof. The linear part of $J$ is clearly continuous; we consider the nonlinear part only. By using the definition of $H$ and Lemma 1, we obtain for $s, t \geq 0$ :

$$
\begin{aligned}
|H(s)-H(t)| & =\left|\int_{t}^{s} h(r) \mathrm{d} r\right|=\left|\int_{t}^{s} \frac{1}{f(r)} \mathrm{d} r\right| \\
& \leq\left|\int_{t}^{s} r^{\frac{1-n}{2 n}} \mathrm{~d} r\right| \leq \frac{2}{p}\left|s^{\frac{p}{2}}-t^{\frac{p}{2}}\right| .
\end{aligned}
$$

Consequently, we obtain for $u, v \in W^{1, p}(\Omega)$ :

$$
\left|\int_{\Omega} H\left(|\nabla u|^{2}\right)-H\left(|\nabla v|^{2}\right) \mathrm{d} x \mathrm{~d} y\right| \leq\left.\frac{2}{p} \int_{\Omega}|| \nabla u\right|^{p}-|\nabla v|^{p} \mid \mathrm{d} x \mathrm{~d} y .
$$

Lemma 4 allows us to conclude.

Theorem 3. Let $\left\{\mathcal{T}_{h}\right\}_{h \in(0,1]}$ be a sequence of refining regular triangulations. Then the sequence of corresponding discrete solutions $\left\{v_{h}\right\}_{h \in(0,1]} \subset W^{1, p}(\Omega)$ weakly converges to the exact solution $v \in W^{1, p}(\Omega)$ as $h$ tends to 0 . Moreover, $\left\{v_{h}\right\}_{h \in(0,1]}$ strongly converges to $v$ in $L^{p}(\Omega)$.

Proof. We prove $\lim _{h \rightarrow 0} v_{h}=v$ by applying Lemma 3. So we consider an arbitrary subsequence $\left\{v_{h_{i}}\right\}_{i \in \mathbb{N}}$ of $\left\{v_{h}\right\}_{h \in(0,1]}$, with $\lim _{i \rightarrow \infty} h_{i}=0$. For each $\mathcal{T}_{h_{i}}$ we define $w_{h_{i}}$, the element of $V_{k, h_{i}}$, which satisfies

$$
\left\|v-w_{h_{i}}\right\|_{W^{1, p}}=\min \left\{\|v-w\|_{W^{1, p}} ; w \in V_{k, h}\right\} .
$$


Now we show that the sequence $\left\{w_{h_{i}}\right\}_{i \in \mathbb{N}}$ is a minimizing sequence for $J$. We know from Lemma 5 that $J$ is continuous. It is then possible for each $\varepsilon>0$ to find $\delta>0$ such that, for each $\tilde{v} \in B(v, \delta)$, we have $|J(v)-J(\tilde{v})| \leq \varepsilon$. It is known that $\left\|v-w_{h_{i}}\right\|_{W^{1, p}}$ tends to 0 as $h_{i}$ tends to 0 . Hence we have established that the sequence $\left\{w_{h_{i}}\right\}_{i \in \mathbb{N}}$ is a minimizing sequence.

The discrete solutions $\left\{v_{h_{i}}\right\}_{i \in \mathbb{N}}$ satisfy $J\left(v_{h_{i}}\right) \leq J\left(w_{h_{i}}\right), \forall i \in \mathbb{N}$. Therefore $\left\{v_{h_{i}}\right\}_{i \in \mathbb{N}}$ is also a minimizing sequence. From the proof of Theorem 1 we know that we can extract a subsequence from $\left\{v_{h_{i}}\right\}_{i \in \mathbb{N}}$ which weakly converges to the exact solution $v$. Lemma 3 let us conclude for the weak convergence in $W^{1, p}(\Omega)$. The compact embedding of $W^{1, p}(\Omega)$ in $L^{p}(\Omega)$ implies that the sequence $\left\{v_{h}\right\}_{h \in(0,1]}$ converges to $v$ strongly.

\section{NUMERICAL EXPERIMENT}

In this section we present a simple numerical algorithm to compute an approximation of the solution of Problem (22). In fact, this algorithm computes an approximation of the solution of the discrete problem (38). The above theory would rather suggest to design a minimization algorithm for the equivalent problem (39). Nevertheless, an algorithm for Problem (38) is certainly easier to design. Therefore in this preliminary numerical investigation we prefer solving the discrete variational problem (38).

Algorithm 1. For solving Problem (38) apply the following steps

a) Choose an initial guess $v_{h}^{(0)}$ for the numerical approximation.

b) For $i=0,1,2, \ldots$ solve the following linear problem: find $v_{h}^{(i+1)} \in V_{k, h}$ satisfying

$$
\int_{\Omega} \frac{1}{f\left(\left|\nabla v_{h}^{(i)}\right|^{2}\right)} \nabla v_{h}^{(i+1)} \nabla w_{h} \mathrm{~d} x \mathrm{~d} y=\frac{1}{2} \int_{\Omega} w_{h} \mathrm{~d} x \mathrm{~d} y, \quad \forall w_{h} \in V_{0, h}
$$

Before considering the numerical convergence, we present a typical solution to Problems $(1-4,6,7)$. We select an example with a small central region at the base $(z=0)$ where we prescribe a Neumann boundary condition; a homogeneous Dirichlet condition is prescribed elsewhere at the base. We take $n=3$, i.e. $p=4 / 3$. The numerical solution is plotted in Figure 1.

We do not provide any theoretical analysis for the convergence of Algorithm 1; we only report observed numerical convergence. A possible numerical experiment consists of applying Algorithm 1 to a modified problem to which we know the exact solution. We do this by a priori choosing a function $\tilde{v} \in W^{1, \frac{4}{3}}(\Omega)$, with $\frac{\partial \tilde{v}}{\partial y}(x, 2)=0$, and then inserting it in the left-hand side of (18). This defines a new right-hand side and consequently a new problem (with adapted boundary conditions and possibly domain). Access to the exact error is then possible for this new problem. It turns out that the convergence rate is very sensitive to the choice of $\tilde{v}$.

Hence, in order to obtain an approximation of the convergence rate for the original problem, we use a sequence of refining grids. The exact problem we consider is : Problem (22) with $T_{0}^{2}=0.1, L=8,((0, L) \times\{0\}) \cap \Gamma_{1}=$ $(3.6,4.4), k(x, 0)=0, k(0, y)=g(L, y)=-\frac{1}{32} y^{4}+\frac{1}{4} y^{3}-\frac{T_{0}^{2}+3}{4} y^{2}+\left(T_{0}^{2}+1\right) y$. (This particular choice for $k$ has a special meaning in glaciology we do not want to explain here.) We estimate the convergence rate by two different means. First, we consider the numerical solution computed on the finest grid as the exact solution and estimate the convergence on much rougher grids. We find:

- Linear convergence for $v$, i.e.

$$
\left\|v-v_{h}\right\|_{L^{\frac{4}{3}(\Omega)}}=\mathcal{O}(h) .
$$

- Sublinear convergence for $\frac{\partial v}{\partial x}$ or more precisely

$$
\left\|\frac{\partial v}{\partial x}-\frac{\partial v_{h}}{\partial x}\right\|_{L^{\frac{4}{3}(\Omega)}}=\mathcal{O}\left(h^{0.7}\right) .
$$



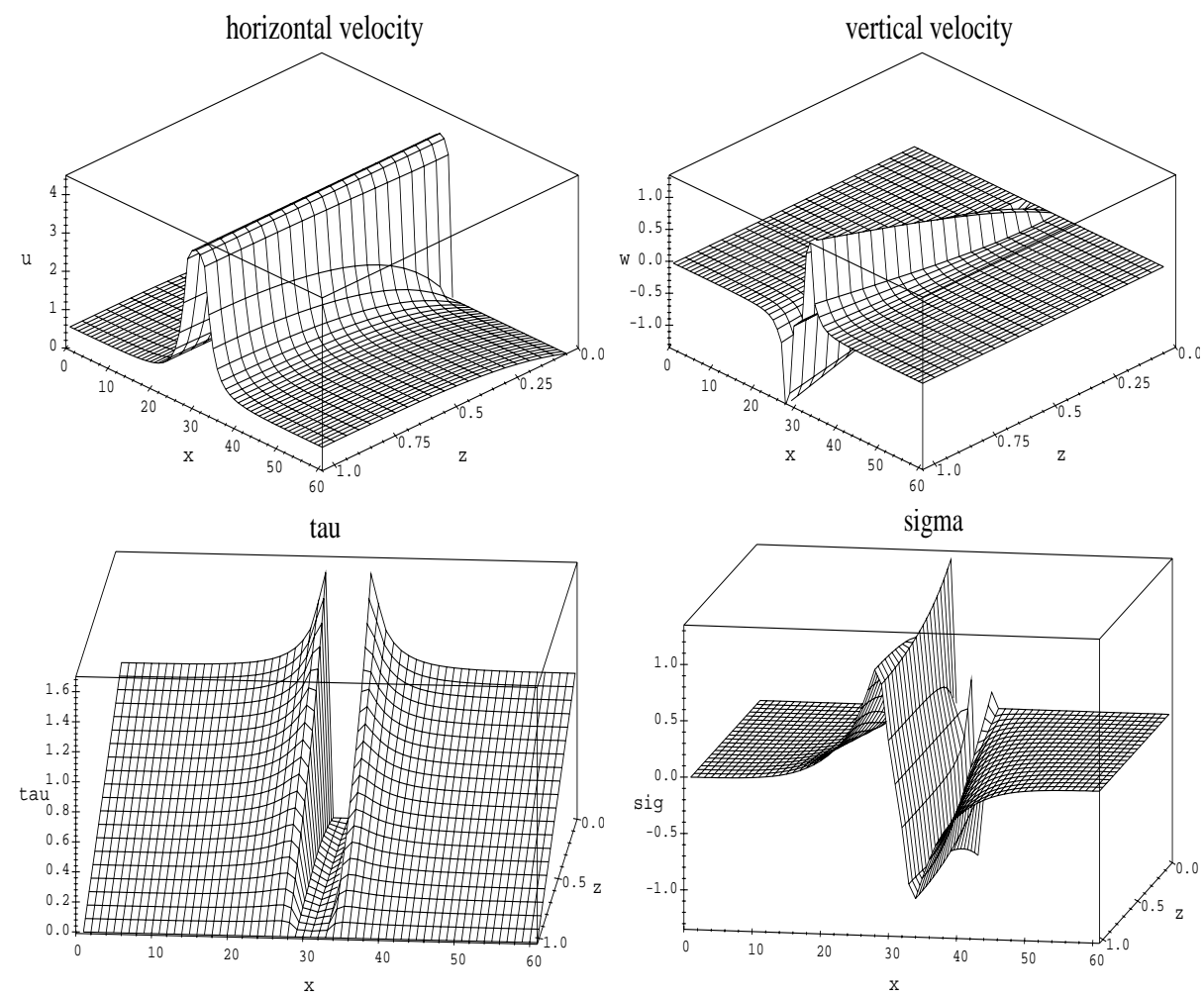

Figure 1. Slab with sliding : $u, w, \tau$ and $\sigma$.

- Sublinear convergence for $\frac{\partial v}{\partial y}$ or more precisely

$$
\left\|\frac{\partial v}{\partial y}-\frac{\partial v_{h}}{\partial y}\right\|_{L^{\frac{4}{3}(\Omega)}}=\mathcal{O}\left(h^{0.8}\right)
$$

A second method which allows us to estimate the convergence rate is the following: we observe that, for any three successive grids $(4 h, 2 h$ and $h)$ in the refining grid sequence, we have

$$
\left\|v_{4 h}-v_{2 h}\right\|_{L^{\frac{4}{3}(\Omega)}}=2\left\|v_{2 h}-v_{h}\right\|_{L^{\frac{4}{3}}(\Omega)} .
$$

We have established convergence of the sequence of discrete solutions, see Theorem 3 . Therefore, for a chosen $\varepsilon>0$, there exists $h_{0} \in(0,1]$ such that

$$
\left\|v-v_{h}\right\|_{L^{\frac{4}{3}(\Omega)}} \leq \varepsilon, \forall h \leq h_{0} .
$$

By applying the triangle inequality, when $h \gg h_{0}$, we find:

$$
\left\|v-v_{h}\right\|_{L^{\frac{4}{3}(\Omega)}} \leq\left\|v-v_{h_{0}}\right\|_{L^{\frac{4}{3}(\Omega)}}+\left\|v_{h_{0}}-v_{2 h_{0}}\right\|_{L^{\frac{4}{3}(\Omega)}}+\cdots+\left\|v_{\frac{h}{2}}-v_{h}\right\|_{L^{\frac{4}{3}}(\Omega)} .
$$


The observation (40) allows us to write this last inequality as

$$
\begin{aligned}
\left\|v-v_{h}\right\|_{L^{\frac{4}{3}}(\Omega)} & \leq \varepsilon+\left(1+\frac{1}{2}+\frac{1}{4}+\cdots\right)\left\|v_{\frac{h}{2}}-v_{h}\right\|_{L^{\frac{4}{3}}(\Omega)} \\
& =\varepsilon+2\left\|v_{\frac{h}{2}}-v_{h}\right\|_{L^{\frac{4}{3}}(\Omega)} .
\end{aligned}
$$

By using (40) once more and (41) we find

$$
\begin{aligned}
\left\|v-v_{h}\right\|_{L^{\frac{4}{3}}(\Omega)} & \leq \varepsilon+2\left\|v_{\frac{h}{2}}-v_{h}\right\|_{L^{\frac{4}{3}}(\Omega)} \\
& =\varepsilon+\frac{1}{2^{k-1}}\left\|v_{2^{k-1} h}-v_{2^{k} h}\right\|_{L^{\frac{4}{3}}(\Omega)}, k \in \mathbb{N}
\end{aligned}
$$

Since $\varepsilon$ is arbitrarily small, we have linear convergence for $v$.

The same argument can be repeated for the derivatives of $v$ because we observe

$$
\begin{aligned}
& \left\|\frac{\partial v_{4 h}}{\partial x}-\frac{\partial v_{2 h}}{\partial x}\right\|_{L^{\frac{4}{3}}(\Omega)}=1.61\left\|\frac{\partial v_{2 h}}{\partial x}-\frac{\partial v_{h}}{\partial x}\right\|_{L^{\frac{4}{3}}(\Omega)} \\
& \left\|\frac{\partial v_{4 h}}{\partial y}-\frac{\partial v_{2 h}}{\partial y}\right\|_{L^{\frac{4}{3}}(\Omega)}=1.77\left\|\frac{\partial v_{2 h}}{\partial y}-\frac{\partial v_{h}}{\partial y}\right\|_{L^{\frac{4}{3}}(\Omega)}
\end{aligned}
$$

for any $h$ in the refining sequence. We find $\left\|\frac{\partial v}{\partial x}-\frac{\partial v_{h}}{\partial x}\right\|_{L^{\frac{4}{3}(\Omega)}}=\mathcal{O}\left(h^{0.7}\right)$ and $\left\|\frac{\partial v}{\partial y}-\frac{\partial v_{h}}{\partial y}\right\|_{L^{\frac{4}{3}}(\Omega)}=\mathcal{O}\left(h^{0.8}\right)$. This confirms what we already found.

If we estimate the convergence rate for the original variables $\tau$ and $\sigma$, instead of the derivatives of $v$, we find an almost linear convergence in $L^{p}(\Lambda)$.

A last aspect we would like to report is the convergence of Algorithm 1 in terms of approximating the exact solution of the discrete problem (38). We observe a linear convergence. At each iteration the difference $\left\|v_{h}^{(i)}-v_{h}^{(i+1)}\right\|_{\infty}$ is divided by about 1.75 . This number seems not to be problem dependent. We also have that, for a given problem, the number of iterations does not change as the grid is refined.

Finally, the convergence rates reported above only change a little if we take different values for $n$ (we tested $n=1,2,3,4,5)$, and overrelaxation reduces the number of iterations by about $30 \%$ for $n=3$.

\section{CONClusions AND FUture WORK}

We have proved the existence and uniqueness of the solution to a nonlinear problem coming from the field of glaciology. We have also proved that the discrete solution converges to the exact solution. A first simple numerical scheme has been presented and tested with reasonable performances. The grid size for the proposed algorithm can be made much finer than what was possible with the previously used techniques in glaciology (see $[1,2,4,5])$.

Several aspects of the work presented in this paper may certainly be improved or completed. A mixed formulation of the discrete problem or finite volume methods should provide more precision in the approximation of $\nabla v$, which is of prime importance to the glaciologist. The convergence rate of Algorithm 1 is only linear and an improvement would be desirable. From a theoretical point of view, a convergence theory for the numerical algorithm and a posteriori error estimates (adaptative grid refinement) are two aspects we would like to develop in order to complete this work.

The authors would like to thank Henri-Michel Maire for Lemma 4. 


\section{REFERENCES}

[1] H. Blatter, Velocity and stress fields in grounded glacier: a simple algorithm for including deviatoric stress gradients. J. Glaciol. 41 (1995) 333-344.

[2] H. Blatter, G.K.C. Clarke and J. Colinge, Stress and velocity fields in glaciers: Part II. Sliding and basal stress distribution. J. Glaciol. (to appear).

[3] P.G. Ciarlet, The Finite Element Method for Elliptic Problems. North-Holland, Amsterdam (1978).

[4] J. Colinge and H. Blatter, Stress and velocity fields in glaciers: Part I. Finite difference schemes for higher order glacier models. J. Glaciol. (to appear).

[5] J. Colinge, Ice mass modelling with shooting techniques (in preparation).

[6] B. Dacorogna, Direct Methods in the Calculus of Variations. Springer, Berlin (1989).

[7] P. Marcellini, Approximation of quasiconvex functions, and lower semi-continuity of multiple integrals. Manuscripta Math. 51 (1985) $1-28$

[8] J. Nečas, Les Méthodes Directes en Théorie des Équations Élliptiques. Masson, Paris (1967).

[9] W.S.B. Paterson, The Physics of Glaciers, 3rd ed. Pergamon/Elsevier, New-York (1994). 